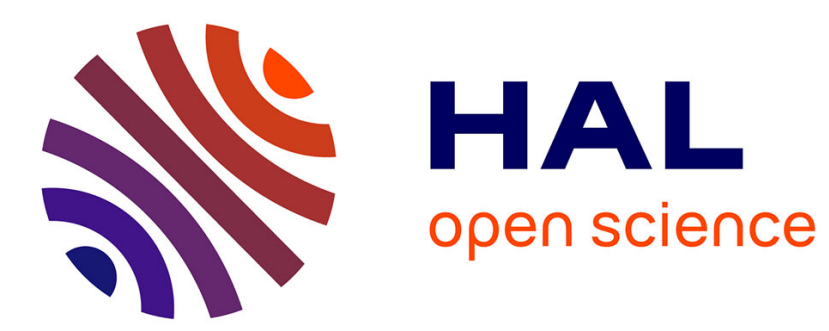

\title{
On the use of XFEM within the Arlequin framework for the simulation of crack propagation
}

\author{
Hachmi Ben Dhia, Olivier Jamond
}

\section{To cite this version:}

Hachmi Ben Dhia, Olivier Jamond. On the use of XFEM within the Arlequin framework for the simulation of crack propagation. 2009. hal-00422595

\section{HAL Id: hal-00422595 \\ https://hal.science/hal-00422595}

Preprint submitted on 7 Oct 2009

HAL is a multi-disciplinary open access archive for the deposit and dissemination of scientific research documents, whether they are published or not. The documents may come from teaching and research institutions in France or abroad, or from public or private research centers.
L'archive ouverte pluridisciplinaire HAL, est destinée au dépôt et à la diffusion de documents scientifiques de niveau recherche, publiés ou non, émanant des établissements d'enseignement et de recherche français ou étrangers, des laboratoires publics ou privés. 


\title{
On the use of XFEM within the Arlequin Framework for the Simulation of Crack Propagation
}

\author{
Hachmi Ben Dhia, Olivier Jamond \\ Ecole Centrale Paris, MSS-MAT, UMR 8579 CNRS, 92295 Chatenay Malabry \\ Cedex France
}

\begin{abstract}
The Arlequin method is a generic numerical method that allows, by local superposition and coupling of models, to address multimodel and multiscale mechanical problems. In particular, this method has already been used to super-impose cracked patches on sound structures, reducing this way the global simulation ressources a classical finite element approach would have required.

In this paper, one of the key features of the Extended-Finite Element Method, namely the Heaviside enrichment function, is used within the Arlequin framework to further reduce the costs of crack propagation simulations. The main goal of the paper is to describe the proposed methodology and to assess its performance through numerical experiments.
\end{abstract}

Key words: multimodel, Arlequin method, XFEM, crack propagation.

\section{Introduction}

Generation of classical regular finite element meshes that are capable of properly representing material discontinuities or accurately simulating the propagation of cracks in complex structures with high strain and stress localization is a tedious and time consuming endeavor. This explains the search over the last two decades for flexible numerical methodologies in order to efficiently tackle this difficult issue. Among others, methods that have been proposed are the so-called meshless methods, see e.g. [30,5], the partition-of-unity method

Email address: hachmi.ben-dhia@ecp.fr, olivier.jamond@ecp.fr (Hachmi Ben Dhia, Olivier Jamond). 
(PUM) $[28,2]$ and its well-known instances, namely the generalized finite element method (GFEM) (e.g. [4]) and the extended finite element method (XFEM), see e.g. $[6,29,20])$. In these methods, somewhat reminiscent of the Treftz approach, the finite element space associated with a given coarse mesh is enriched by analytical or semi-analytical functions based on a priori knowledge of the unknown solution. In the XFEM approach, for example, one uses level set functions and asymptotic local modal functions around the cracks.

XFEM/GFEM methods are particularly relevant and effective whenever the local behavior of the solution is known.

Another class of approaches is given by the s-method, which is in many ways similar to the Fast Adaptive Composite-grid (FAC) method [27]. This approach can be considered as a genuine finite element method in which the coarse finite element space is locally enriched by fine scale basis functions associated with a fine mesh. Although effective in many regards, the method may suffer from redundancy. Moreover, the resulting problem in the s-method is solved globally. An approach similar to the s-method is one that involves the use of local meshes (also called patches), but whose resulting system of equations is solved by a relaxed Chimera algorithm [23,24] (see also the Schwarz algorithm (see e.g [37])).

Our ultimate goal being the simulation of physically-based damage, localisations and crack propagation, we propose a novel and alternative approach, experienced in this paper on basic tests. The approach is based on the multimodel and multiscale Arlequin framework $[8,10,33,11,12,16]$. The method essentially consists in superimposing a fine scale model (in a patch) to a large scale model combined together using a partition of energy. The construction of the resulting coupled model allows for great flexibility; indeed, one may independently refine the mesh or use different constitutive laws, governing equations, or geometries within the patch. For instance, the Arlequin framework has been recently employed for successfully coupling atomistic models with continuum models [38,15,3,32].

The idea of using the Arlequin framework for the simulation of crack propagation, fatigue, or damage phenomena in a structure, has already been advanced a few years ago $[34,13,35]$. In order to expose our motivation and put in evidence our new numerical achievements, we report here some of our earlier results. In the first example (from [34]), we considered a solid composed of two materials, one soft (rubber) and one hard (steel). The structure was submitted to a cyclic loading on the top surface, to symmetry conditions along the bottom surface, while the other two boundaries were kept free. Figure 1 shows the evolution of damage as well as nucleation and propagation of macroscopic cracks whenever damage exceeded a prescribed threshold. 


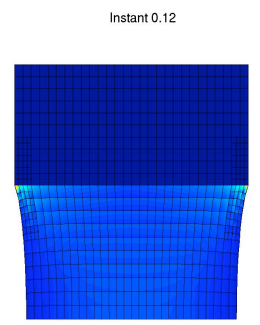

(a)

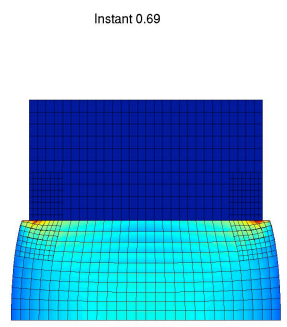

(b)

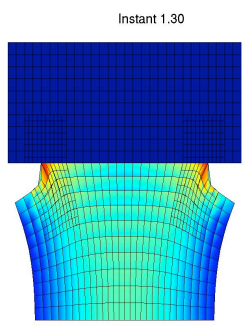

(c)

Fig. 1. Propagation of damage and creation of a crack within the Arlequin framework (three selected snapshots)

The fine scale phenomena near the crack tip were taken into account using an Arlequin patch which was free to move and grow according to the evolution of the crack tip. In this case, the simulation was still relatively simple due to the fact that the direction of propagation was a priori known to be the straight bi-material interface. The second example, taken from [35], illustrates the crack propagation in a linearly elastic structure as shown in Figure 2.

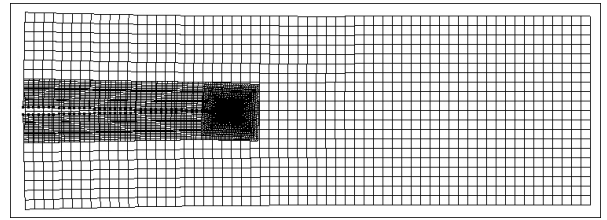

(a)

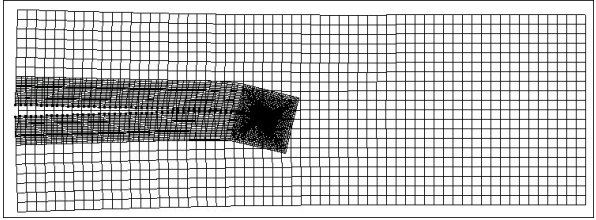

(b)

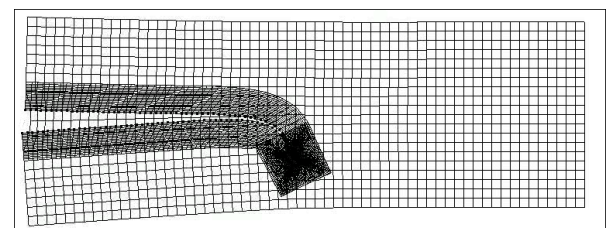

(c)

Fig. 2. Propagation of a crack for the double-cantilever elastic beam (three selected snapshots)

The patch consisted of a very fine mesh at the tip and was progressively adapted following the evolution of the crack. We note that the underlying coarse mesh was kept unchanged during the whole process. Data for this test case (referred to as the double cantilever beam test) was taken from Belytschko and Black [6]. The obvious drawback of the method was that the number of finite elements along the lips of the crack was far from being optimal, which unnecessarily increased the cost of the computation. Moreover, the treatment of more than one crack would quickly become an intricate issue.

In this work, we propose to circumvent the aforementioned issues by intro- 
ducing Heaviside enrichment functions, as in XFEM, within our Arlequin framework, with the objective of further reducing computational costs and enhancing the flexibility of crack propagation simulations in this framework. In particular, we will show that the combined approach allows us to consider smaller patches to capture the local features near the tip since Heaviside functions are used to enrich the coarse model. The main goal of the present paper is to describe the proposed methodology and to assess its performance through numerical experiments.

The paper is organized as follows. Following the introduction, we provide in Section 2 the continuous formulation of the crack problem in a linearly elastic structure. In Section 3, we recall the main features of the extended finite element method. We then show in Section 4 how enrichment functions a la XFEM can be incorporated into our Arlequin framework. In particular, we describe the continuous Arlequin formulation for the crack problem in Subsection 4.1. The discrete counterpart is given in Subsection 4.2 and practical aspects related to numerical integration are detailed in Subsection 4.3. Numerical results in order to assess the performance of our approach are then presented in Section 5. We use to that effect two examples, one dealing with the simulation of a kinked crack and the double-cantilever beam test case. In these two examples, the patch is strictly contained in the interior of the global domain (no intersection between the boundary of the patch and the global domain boundary). A third numerical example shows the feasibility of the simulation of the propagation of a crack from a side of a square plate to the opposite one. This example shows, in particular, how the patch is adapted easily when it hurts the boundary of the global domain. The paper closes with concluding remarks and some ongoing works in Section 6.

\section{Formulation of a cracked elastic body problem}

Let us consider a linear elastic cracked solid, occupying the closure of a bounded domain $\Omega_{0}$. For the sake of clarity, we will consider the bidimensional case. The boundary of the solid is partitioned into $\Gamma_{u}, \Gamma_{g}, \Gamma^{+}$and $\Gamma^{-}$, where $\Gamma_{u}$ is the part where the solid is clamped and $\Gamma_{g}$ is the part on which an applied density of loads $\boldsymbol{g}$ is given. The other two parts of the boundary $\Gamma^{+}$ and $\Gamma^{-}$define the faces of the crack whose tip is denoted by $\boldsymbol{o}$ (see figure 3 ). It is assumed that the crack is closed in the undeformed configuration of the solid. Thus, from a geometrical point of view, we have $\Gamma^{+} \equiv \Gamma^{-} \equiv \Gamma$. But free boundary conditions are applied on $\Gamma^{+}$and $\Gamma^{-}$in the remainder of the article. That is to mean that no unilateral contact loads are considered between the

faces of the crack. Moreover, for the sake of conciseness, we neglect external body forces.

The classical weak form of the linearized primal elastic problem reads: 


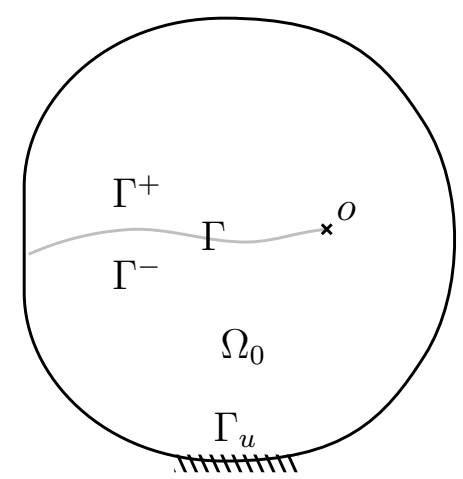

Fig. 3. problem of a cracked linear elastic solid

$$
\begin{gathered}
\text { find } \boldsymbol{u} \in \boldsymbol{V}_{0} ; \forall \boldsymbol{v} \in \boldsymbol{V}_{0} \\
\qquad a(\boldsymbol{u}, \boldsymbol{v})=l(\boldsymbol{v})
\end{gathered}
$$

where:

$$
\begin{gathered}
\boldsymbol{V}_{0}=\left\{\mathbf{v} \in \boldsymbol{H}^{1}\left(\Omega_{0}\right) ; \boldsymbol{v}=0 \text { on } \Gamma_{u}\right\} \\
a(\boldsymbol{u}, \boldsymbol{v})=\int_{\Omega_{0}} \sigma(\boldsymbol{u}): \epsilon(\boldsymbol{v}) d x \\
l(\boldsymbol{v})=\int_{\Gamma_{g}} \boldsymbol{g} \cdot \boldsymbol{v} d \gamma
\end{gathered}
$$

with $\sigma$ and $\epsilon$ standing for the stress and linearized strain tensors, the stess tensor being related to $\epsilon(\boldsymbol{u})=\nabla_{s}(\boldsymbol{u})$ by the Hooke's law:

$$
\sigma_{i j}(\boldsymbol{u})=\boldsymbol{R}_{i j k l} \epsilon_{k l}(\boldsymbol{u}) \quad \text { in } \Omega_{0}
$$

where the Einstein's convention of summation on repeated indices is used and where the elasticity tensor $\boldsymbol{R}$ is assumed to verify:

$$
\begin{gathered}
\boldsymbol{R}_{i j k l}=\boldsymbol{R}_{j i k l}=\boldsymbol{R}_{k l i j} \quad \text { (symmetry) } \\
\exists c_{0} ; \quad \boldsymbol{R}_{i j k l} \tau_{i j} \tau_{k l} \geq c_{0} \tau_{i j} \tau_{i j}, \quad \forall \tau_{i j}=\tau_{j i} \quad \text { (coercivity) } \\
\boldsymbol{R}_{i j k l} \in \boldsymbol{L}^{\infty}\left(\Omega_{0}\right) \quad \text { (regularity) }
\end{gathered}
$$

For a classical conforming finite element approximation of this problem, the mesh has to match the crack. But since the latter may propagate into the solid without necessarily fiting the existing mesh edges, one has to generate repetively new meshes compatible with the evolution of the crack in order to achieve an accurate numerical approximation of the problem solution. This procedure is clearly unflexible and costly. The Extended Finite Element Method is one of the most efficient methods that has been designed to address this issue. Since the XFEM is partly used in our approach, its main features are recalled in the next section where some notations are introduced. 


\section{XFEM discretisation}

The XFEM discretization of the problem (1)-(5) consits in constructing a finite dimensional subspace of $\boldsymbol{V}_{\mathbf{0}}$ by enrichment of a classical finite element subspace of

$$
\boldsymbol{V}_{0}^{\Gamma}=\left\{\boldsymbol{v} \in \boldsymbol{H}^{1}\left(\Omega_{0}^{\Gamma}\right) ; \boldsymbol{v}=0 \text { in } \Gamma_{u}\right\}
$$

where $\Omega_{0}^{\Gamma}=\Omega_{0} \cup \Gamma$ denotes the uncracked domain.

This is done as follows. Let $T_{h}$ be a mesh of the uncracked solid whose edges do not necessarily match $\Gamma$. Let $\left(\boldsymbol{\varphi}_{1}, \boldsymbol{\varphi}_{2}, \ldots, \boldsymbol{\varphi}_{n}\right)$ denote a basis spanning a classical Lagrange finite element space $\boldsymbol{V}_{0 h}^{\Gamma}$, associated to $T_{h}$ (see e.g. $[19,39,25]$ ) and assumed to be a subspace of $\boldsymbol{V}_{0}^{\Gamma}$. The compact support of a shape function $\boldsymbol{\varphi}_{i}$ is denoted by $\omega_{i}$ and its interior by $\stackrel{\circ}{\omega}_{i}$. The set of indices $k$ such that $\boldsymbol{o} \in \omega_{k}$ is denoted by $K^{s}\left(K^{s}=\{7,8,9,10\}\right.$ in the scalar case depicted by figure 4$)$. The set of indices $k$ such that $k \notin K^{s}$ and $\stackrel{\circ}{\omega}_{k} \cap \Gamma \neq \emptyset$ is denoted by $K^{d}\left(K^{d}=\{1,2,3,4,5,6\}\right.$ in figure 4$)$. The union of $\omega_{k}, k \in K^{d}$, defines a domain we denote by $\Omega_{d}$. The latter is divided by the crack $\Gamma$ into two domains, denoted by $\Omega_{d}^{+}$and $\Omega_{d}^{-}$. A precise definition of the domain $\Omega_{d}^{+}$(and thus of $\Omega_{d}^{-}$) relies on the signed distance function: by defining at each point $\boldsymbol{p} \in \Gamma$ a classical positively oriented frame $\left(\boldsymbol{\tau}_{p}, \boldsymbol{\nu}_{p}\right)$ where $\boldsymbol{\tau}_{p}$ and $\boldsymbol{\nu}_{p}$ refer to unit tangent and normal vectors to the crack at point $\boldsymbol{p}$ (see figure 5), one can associate to each point $\boldsymbol{x} \in \Omega_{d}$ (one of) its nearest point(s) $\boldsymbol{x}^{*}$ on $\Gamma$ (as in contact mechanics, e.g. [14]). The domain $\Omega_{d}^{+}$is then defined by: (see figure 5)

$$
\Omega_{d}^{+}=\left\{\boldsymbol{x} \in \Omega_{d} ;\left(\boldsymbol{x}-\boldsymbol{x}^{*}\right) \cdot \boldsymbol{\nu}_{x^{*}} \geq 0\right\}
$$

The XFEM finite dimensional space is then defined by:

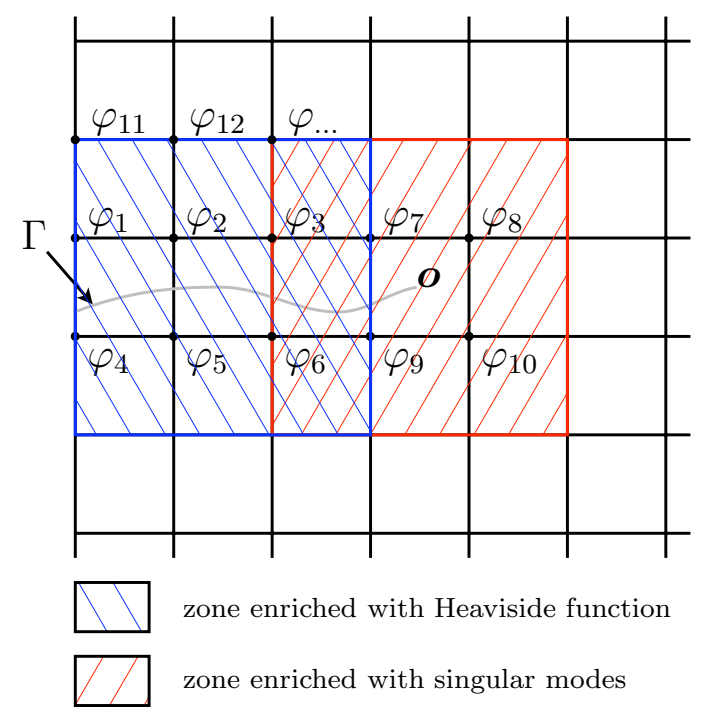

Fig. 4. XFEM discretization

$$
\boldsymbol{V}_{0 h}=\operatorname{span}\left\{\left(\boldsymbol{\varphi}_{i}\right)_{i=1, n},\left(H \boldsymbol{\varphi}_{j}\right)_{j \in K^{d}},\left(F_{k} \boldsymbol{\varphi}_{l}\right)_{k=1,4 ; l \in K^{s}}\right\}
$$




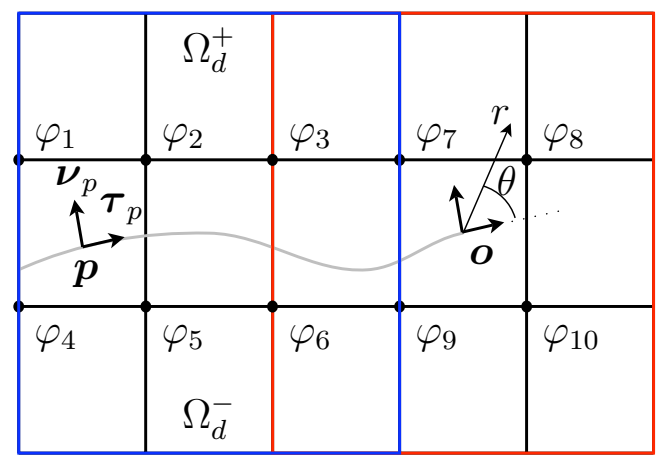

Fig. 5. local frame

where $H$ refers to the sign-like field, defined by:

$$
H(x)=\left\{\begin{array}{l}
+1 \text { in } \Omega_{d}^{+} \\
-1 \text { elsewhere }
\end{array}\right.
$$

and where the functions $F_{j}$ are given by:(see e.g.[6,29,20,26,18], for details and more general considerations)

$$
\left\{F_{j}(r, \theta), j=1,4\right\}=\left\{\sqrt{r} \sin \frac{\theta}{2}, \sqrt{r} \cos \frac{\theta}{2}, \sqrt{r} \sin \frac{\theta}{2} \sin \theta, \sqrt{r} \cos \frac{\theta}{2} \sin \theta\right\}
$$

with $(r, \theta)$ referring to the polar coordinates in the local frame $\left(\boldsymbol{o}, \boldsymbol{\tau}_{o}, \boldsymbol{\nu}_{o}\right)$ (see figure 5). Observe here that the enriched XFEM solution is defined by:

$$
\boldsymbol{u}_{h 0}=\sum_{i=1, n} u_{i} \boldsymbol{\phi}_{i}+\sum_{j \in K_{d}} a_{j} H \boldsymbol{\phi}_{j}+\sum_{k=1,4} \sum_{l \in K^{s}} b_{l}^{k} F_{k} \boldsymbol{\phi}_{l}
$$

where $\left(u_{i}\right)_{i=1, n},\left(a_{j}\right)_{j \in K_{d}}$ and $\left(b_{l}^{k}\right)_{k=1,4 ; l \in K^{s}}$ are the unknown degrees of freedom.

To summarize, for the simulation of a cracked solid, XFEM is based on a double enrichment of classical finite element spaces by:

- a localized sign-like field that reproduces the material discontinuity due to the crack;

- localized asymptotic modes in the near crack tip zone.

The first enrichment could be considered as universal. In the contrary, for general mechanical problems, the asymptotic knowledge of the behaviour of the solution in critical zones is unfortunately unavailable and one has to resort to numerical approximations in these zones. For the numerical treatment of these general problems, we suggest here to use only the first enrichment within the Arlequin framework. 


\section{Use of XFEM within the Arlequin framework}

In this section, by using the Arlequin framework, a local model is superimposed to the global one in the neighbourhood of the crack tip (see figure 6 ). The two models are combined together using a partition of energy and a coupling operator, leading to a continuous Arlequin formulation of the problem, detailed in Subsection 4.1.

A first key (modeling) feature of this continuous formulation is that it al-

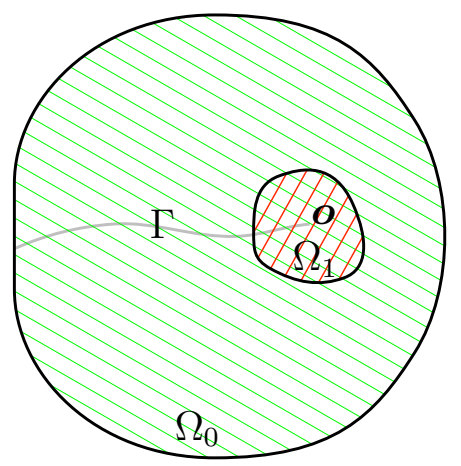

Fig. 6. Arlequin domains

lows us, by means of different approximations of the super-imposed models, to elaborate multimodel (and also multiscale) discrete formulations. The one elaborated in Subsection 4.2 uses: (see figure 7)

- the XFEM to enrich a coarse global finite element approximation of the global model;

- a fine finite element space for the approximation of the local model. (Observe here that discrete models such as atomistic ones can also be used locally instead of the continuum elasticity model [38,15,3,32], but these topics are not considered in this paper).

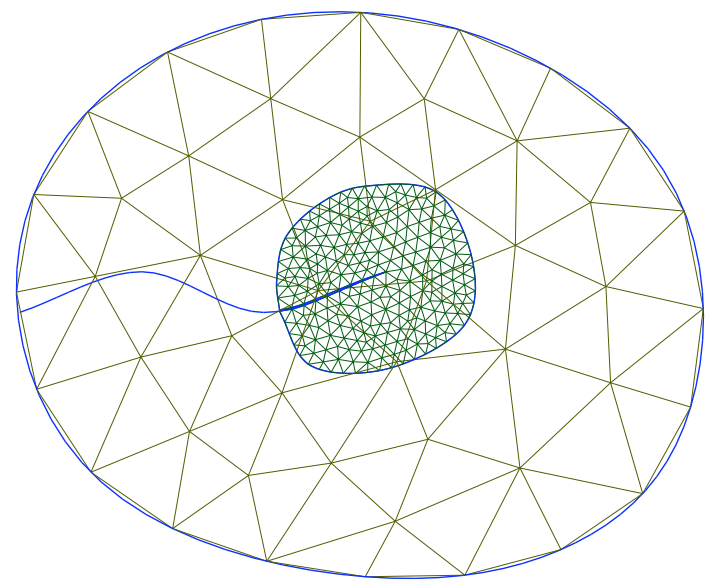

Fig. 7. Examples of meshes for the two arlequin models 
A second key (computational) feature of the Arlequin formulation is related to its substructuring character which allows for the use of domain decompositionlike solvers (see e.g. [21,17]).

\subsection{Continuous Arlequin Formulation}

Let $\Omega_{1}$ be a (copy of a) non-zero measured given regular subdomain of $\Omega_{0}$, located in the near crack tip zone. We assume for clarity that $\Omega_{1}$ is strictly embedded in $\Omega_{0}$ (see figure 6). This subdomain is partitioned into two regular non overlapping domains $\Omega_{c}$ and $\Omega_{f}$ such that (see figure 8):

$$
\begin{gathered}
\boldsymbol{o} \in \Omega_{f} \\
\partial \Omega_{1} \subset \partial \Omega_{c} \\
\operatorname{meas}\left(\Omega_{i}\right)>0, i=c, f
\end{gathered}
$$

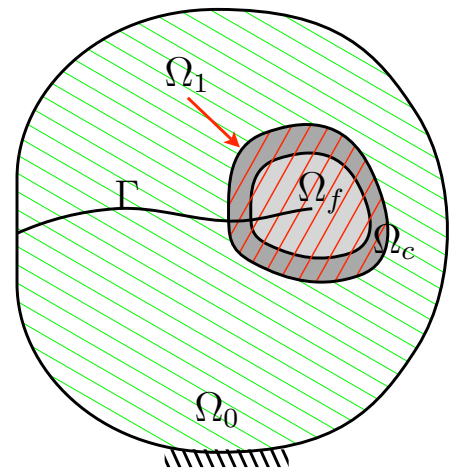

Fig. 8. Arlequin domains

The domains $\Omega_{c}$ and $\Omega_{f}$ are the models coupling and free zones. In the former, the models are coupled; so that they have to be quite similar. In the latter, the models are super-imposed but free from each other; so that they can potentially be very different.

A Lagrangian Arlequin formulation of our problem, using an energy scalar product as coupling operator, reads as following: (see e.g. $[10,11,16]$ )

$$
\begin{gathered}
\text { Find }\left(\boldsymbol{u}_{0}, \boldsymbol{u}_{1}, \boldsymbol{\Phi}\right) \in \boldsymbol{V}_{0} \times \boldsymbol{V}_{1} \times \boldsymbol{M} ; \\
\forall \boldsymbol{v}_{0} \in \boldsymbol{V}_{0}, \quad a_{0}\left(\boldsymbol{u}_{0}, \boldsymbol{v}_{0}\right)+c\left(\boldsymbol{\Phi}, \boldsymbol{v}_{0}\right)=l_{0}\left(\boldsymbol{v}_{0}\right) \\
\forall \boldsymbol{v}_{1} \in \boldsymbol{V}_{1}, \quad a_{1}\left(\boldsymbol{u}_{1}, \boldsymbol{v}_{1}\right)-c\left(\boldsymbol{\Phi}, \boldsymbol{v}_{1}\right)=0 \\
\forall \boldsymbol{\Psi} \in \boldsymbol{M}, \quad c\left(\boldsymbol{\Psi}, \boldsymbol{u}_{0}-\boldsymbol{u}_{1}\right)=0
\end{gathered}
$$

where $\boldsymbol{V}_{0}$ is defined by (2) and where $\boldsymbol{V}_{1}=\boldsymbol{H}^{1}\left(\Omega_{1}\right)$ and the mediator space $\boldsymbol{M}=\boldsymbol{H}^{1}\left(\Omega_{c}\right)$. Furthermore, for $i=0,1$, the weighted internal and external 
virtual works are defined by:

$$
\begin{gathered}
\forall \boldsymbol{u}_{i}, \boldsymbol{v}_{i} \in \boldsymbol{V}_{i}, \quad a_{i}\left(\boldsymbol{u}_{i}, \boldsymbol{v}_{i}\right)=\int_{\Omega_{i}} \alpha_{i} \boldsymbol{\sigma}\left(\boldsymbol{u}_{i}\right): \boldsymbol{\varepsilon}\left(\boldsymbol{v}_{i}\right) d x \\
\forall \boldsymbol{v}_{0} \in \boldsymbol{V}_{0}, \quad l_{0}\left(\boldsymbol{v}_{0}\right)=\int_{\Gamma_{n}} \boldsymbol{g} \cdot \boldsymbol{v}_{0} d \gamma
\end{gathered}
$$

The internal energy weight parameter functions $\left(\alpha_{i}\right), i=0,1$, are defined in th whole domain $\Omega_{0}$ and satisfy: $([12,16])$

$$
\begin{gathered}
\alpha_{i} \in[0,1] \text { in } \Omega_{0} \\
\alpha_{0}+\alpha_{1}=1 \text { in } \Omega_{0} \\
\alpha_{0}=1 \text { in } \Omega_{0} \backslash \Omega_{1} \\
\exists \alpha_{0}>0 ; \alpha_{i} \geq \alpha_{0} \text { in } \Omega_{f}
\end{gathered}
$$

The coupling operator $c$ is defined by:

$$
\forall(\boldsymbol{\psi}, \boldsymbol{v}) \in \boldsymbol{M} \times \boldsymbol{V}_{1}, c(\boldsymbol{\psi}, \boldsymbol{v})=\int_{\Omega_{c}} \kappa_{0}\{\boldsymbol{\psi} \cdot \boldsymbol{v}\}+\kappa_{1}\{\boldsymbol{\epsilon}(\boldsymbol{\psi}): \boldsymbol{\epsilon}(\boldsymbol{v})\} d x
$$

The positive parameters $\kappa_{0}$ and $\kappa_{1}$ are chosen such that the two terms defining $c$ are homogeneous. In the numerical section, we have taken, as in [11], $\kappa_{0}=1$ and $\kappa_{1}=l_{c}^{2}$, where $l_{c}$ refers to the thickness of the coupling zone $\Omega_{c}$.

Before closing this subsection, let us here recall two mathematical results concerning the Arlequin problem, defined above:

- Stability: the mixed Arlequin problem, defined by (19)-(28), has been analyzed mathematically and proved to be well-posed in [16] and previously in [9], but under more stringent conditions on the $\alpha_{i}$ (not allowing for regular $\left.\alpha_{i}\right)$.

- Relevance of the Arlequin formulation: if one modifies, for $\Omega_{0}$, the part of the crack contained in $\Omega_{f}$, without modifying the same part of the crack for the local domain $\Omega_{1}$, then, under some appropriate hypotheses on the data [16]; mainly when, the restriction to $\Omega_{f}$ of $\alpha_{1}$ is a constant that goes to 1 , the solution of the problem (19)-(28) converges to the solution of the (mono-)model problem, defined in Section 2. This result, proved in [16] and assessed by previously published numerical tests (e.g. $[10,11,16])$, is used in the sequel for a flexible construction of the discrete Arlequin problems.

\subsection{Discretisation of the Arlequin problem using XFEM}

To solve efficiently the Arlequin problem defined in the previous subsection, we suggest the following scheme: let $T_{h}^{0}$ be a mesh of the unfractured domain $\Omega_{0}^{\Gamma}$. Let $T_{h}^{1}$ be a mesh of $\Omega_{1}$, significantly finer than $T_{h}^{0}$, especially in the very near crack tip zone. Let $\Omega_{o}^{s}$ denote the set of elements of $T_{h}^{0}$ containing the 
crack tip $\boldsymbol{o}$. Let $\Gamma_{0}$ denote the part of the crack $\Gamma$ exterior to $\Omega_{o}^{s}$. Finally, let $B_{0}=\left(\boldsymbol{\varphi}_{0 i}, i=1, n_{0}\right)$ be a classical vector-valued finite element basis associated to the mesh $T_{h}^{0}$ and denote by $\boldsymbol{V}_{0 h}^{r}$ the finite element space spanned by $B_{0}$.

Referring to XFEM recalled in Section 3, the coarse space $\boldsymbol{V}_{0 h}^{r}$ is enriched as following:

let $K_{0}^{d}$ be the set of indices $k$ such that $\stackrel{\circ}{\omega}_{k} \cap \Gamma_{0} \neq \emptyset$ (recall that $\stackrel{\circ}{\omega}_{k}$ stands for the interior of the support of the basis function $\varphi_{0 k}$ ). Let $\Omega_{d}$ denote the union of these supports and define $\Omega_{d}^{+}$as in (10). The space $\boldsymbol{V}_{0 h}^{r}$ is enriched by the space $\boldsymbol{V}_{0 h}^{d}=\operatorname{span}\left\{H \varphi_{0 j}, j \in K_{0}^{d}\right\}$ where $H$ is the sign field, defined by (12). The following finite dimensional space:

$$
\boldsymbol{V}_{0 h}=\boldsymbol{V}_{0 h}^{r}+\boldsymbol{V}_{0 h}^{d}=\operatorname{span}\left\{\left(\boldsymbol{\varphi}_{0 i}\right)_{i=1, n_{0}},\left(H \boldsymbol{\varphi}_{0 j}\right)_{j \in K_{0}^{d}}\right\}
$$

is then taken as an approximation of $\boldsymbol{V}_{0}$.

Now, based on the fine mesh $T_{h}^{1}$ of the cracked subdomain $\Omega_{1}$, a classical finite element subspace of $\boldsymbol{V}_{1}$, denoted $\boldsymbol{V}_{1 h}$, is constructed. The discrete mediator space, denoted $\boldsymbol{M}_{h}$, is taken as the restriction of $\boldsymbol{V}_{1 h}$ to the coupling zone $\Omega_{c}$ (though other choices are possible, as reported in $[11,16]$ ). Finally, when using low order finite elements to generate the coarse spaces $\boldsymbol{V}_{0 h}$, we assume that:

$$
\Omega_{o}^{s} \subset \Omega_{f}
$$

The condition (30) is a kind of relaxation of the condition (15). It takes into account the finite scale introduced by the coarse discretization and means that the free zone $\Omega_{f}$ has at least to contain the coarse elements that contain the singular crack tip. Its optimality in the discrete finite element framework as a low bound for the size of $\Omega_{f}$ is assessed in the numerical Section (see also [17] for other investigations on this topic).

With these finite dimensional spaces, our discrete enriched finite element approximation of the problem defined by (19)-(28), using XFEM, reads as follows.

$$
\begin{gathered}
\operatorname{Find}\left(\boldsymbol{u}_{0 h}^{r}, \boldsymbol{u}_{0 h}^{d}, \boldsymbol{u}_{1 h}, \boldsymbol{\phi}_{h}\right) \in \boldsymbol{V}_{0 h}^{r} \times \boldsymbol{V}_{0 h}^{d} \times \boldsymbol{V}_{1 h} \times \boldsymbol{M}_{h} \\
\forall\left(\boldsymbol{v}_{0 h}^{r}, \boldsymbol{v}_{0 h}^{d}, \boldsymbol{v}_{1 h}, \boldsymbol{\psi}_{h}\right) \in \boldsymbol{V}_{0 h}^{r} \times \boldsymbol{V}_{0 h}^{d} \times \boldsymbol{V}_{1 h} \times \boldsymbol{M}_{h} \\
a_{0}\left(\boldsymbol{u}_{0 h}^{r}, \boldsymbol{v}_{0 h}^{r}\right)+a_{0}^{d}\left(\boldsymbol{u}_{0 h}^{d}, \boldsymbol{v}_{0 h}^{r}\right)+c\left(\boldsymbol{\phi}_{h}, \boldsymbol{v}_{0 h}^{r}\right)=l_{0}\left(\boldsymbol{v}_{0 h}^{r}\right) \\
a_{0}^{d}\left(\boldsymbol{u}_{0 h}^{r}, \boldsymbol{v}_{0 h}^{d}\right)+a_{0}\left(\boldsymbol{u}_{0 h}^{d}, \boldsymbol{v}_{0 h}^{d}\right)+c^{d}\left(\boldsymbol{\phi}_{h}, \boldsymbol{v}_{0 h}^{d}\right)=l_{0}\left(\boldsymbol{v}_{0 h}^{d}\right) \\
a_{1}\left(\boldsymbol{u}_{1 h}, \boldsymbol{v}_{1 h}\right)-c\left(\boldsymbol{\phi}_{h}, \boldsymbol{v}_{1 h}\right)=0 \\
c\left(\boldsymbol{\psi}_{h}, \boldsymbol{u}_{0 h}^{r}-\boldsymbol{u}_{1 h}\right)+c^{d}\left(\boldsymbol{\psi}_{h}, \boldsymbol{u}_{0 h}^{d}\right)=0
\end{gathered}
$$


where:

$$
\begin{aligned}
a_{0}^{d}\left(\boldsymbol{u}_{0 h}^{r}, \boldsymbol{v}_{0 h}^{d}\right)= & \int_{\Omega_{0} \cap \Omega_{d}^{+}} \alpha_{0} \boldsymbol{\sigma}\left(\boldsymbol{u}_{0 h}^{r}\right): \boldsymbol{\varepsilon}\left(\boldsymbol{v}_{0 h}^{d}\right) d x+\int_{\Omega_{0} \cap \Omega_{d}^{-}} \alpha_{0} \boldsymbol{\sigma}\left(\boldsymbol{u}_{0 h}^{r}\right): \boldsymbol{\varepsilon}\left(\boldsymbol{v}_{0 h}^{d}\right) d x \\
c^{d}\left(\boldsymbol{\psi}_{h}, \boldsymbol{u}_{0 h}^{d}\right)= & \left.\left.\int_{\Omega_{c} \cap \Omega_{d}^{+}}\left\{\kappa_{0} \boldsymbol{\psi}_{h} \cdot \boldsymbol{u}_{0 h}^{d}\right)+\kappa_{1} \boldsymbol{\epsilon}\left(\boldsymbol{\psi}_{h}\right): \boldsymbol{\epsilon}\left(\boldsymbol{u}_{0 h}^{d}\right)\right)\right\} d x \\
& \left.\left.+\int_{\Omega_{c} \cap \Omega_{d}^{-}}\left\{\kappa_{0} \boldsymbol{\psi}_{h} \cdot \boldsymbol{u}_{0 h}^{d}\right)+\kappa_{1} \boldsymbol{\epsilon}\left(\boldsymbol{\psi}_{h}\right): \boldsymbol{\epsilon}\left(\boldsymbol{u}_{0 h}^{d}\right)\right)\right\} d x
\end{aligned}
$$

As a matter of fact, one can observe that, unless the crack tip $\boldsymbol{o}$ belongs to the boundary $\partial \Omega_{d}$, the space $\boldsymbol{V}_{0 h}$ is not a subspace of $\boldsymbol{V}_{0}$. Indeed, assume that the crack tip $\boldsymbol{o} \notin \partial \Omega_{d}$ (see figure 9). Under this hypothesis, the set $\Omega_{o}^{s}$ is necessarily composed of only one element of $T_{h}^{0}$. Consider now the subset of basis functions $H \boldsymbol{\varphi}_{0 k}$ such that $\Omega_{o}^{s}$ is included in the support $\omega_{k}$ of $\boldsymbol{\varphi}_{0 k}$. These functions are clearly discontinuous, not only on the part of the crack contained in $\Omega_{o}^{s}$, but also on its fictive straight extension along the tangent vector $\boldsymbol{\tau}_{o}$ from the crack tip $\boldsymbol{o}$ till the point $\boldsymbol{o}^{\prime}$ where the extension crosses the boundary of $\Omega_{o}^{s}$ (see figure 9). Thus, the discrete problem, defined by (31)-(33), is a nonconforming approximation of the continuous problem (19)-(28). However, and this is one of the main features of the Arlequin method recalled at the end of the previous subsection, this non-conformity is with no significant impact on the solution when the Arlequin parameters are chosen appropriately: in the considered scenario, with a sufficienly large free zone, one has simply to take the weight parameter $\alpha_{1}$ constant and approximately equal to one in $\Omega_{f}$ to stress the patch model and compute a good Arlequin solution.

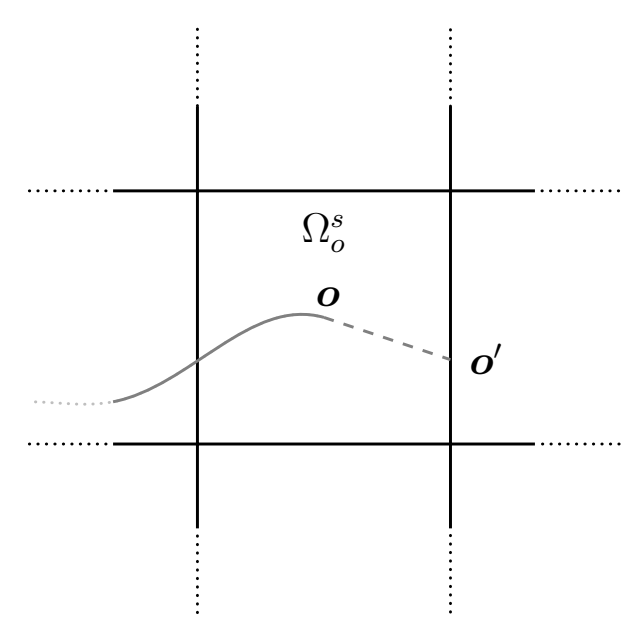

Fig. 9. Element containing the crack tip 


\subsection{Some computational aspects}

The discrete problem (31)-(34) is equivalent to the following linear mixed system:

$$
\left(\begin{array}{cccc}
\boldsymbol{K}_{0}^{r r} & \boldsymbol{K}_{0}^{r d} & \mathbf{0} & \boldsymbol{C}_{0}^{r} \\
\boldsymbol{K}_{0}^{r d^{\top}} & \boldsymbol{K}_{0}^{d d} & \mathbf{0} & \boldsymbol{C}_{0}^{d} \\
\mathbf{0} & \mathbf{0} & \boldsymbol{K}_{1} & \boldsymbol{C}_{1} \\
\boldsymbol{C}_{0}^{r \top} & \boldsymbol{C}_{0}^{d^{\top}} & \boldsymbol{C}_{1}^{\top} & \mathbf{0}
\end{array}\right)\left(\begin{array}{c}
\boldsymbol{U}_{0}^{r} \\
\boldsymbol{U}_{0}^{d} \\
\boldsymbol{U}_{1} \\
\Phi
\end{array}\right)=\left(\begin{array}{c}
\boldsymbol{F}_{0}^{r} \\
\boldsymbol{F}_{0}^{d} \\
\boldsymbol{F}_{1} \\
\mathbf{0}
\end{array}\right)
$$

The stiffness matrix $\boldsymbol{K}_{0}^{r d}$ involves terms that require specific numerical integration, when compared to the ones developed for the standard Arlequin formulation, given in [11] and for the XFEM, given in [29]. Indeed, the generic terms of $\boldsymbol{K}_{0}^{r d}$ read:

$$
\left(\boldsymbol{K}_{0}^{r d}\right)_{i j}=a_{0}^{d}\left(\boldsymbol{\varphi}_{0 i}, \boldsymbol{\varphi}_{0 j}^{d}\right)
$$

where $\boldsymbol{\varphi}_{0 i}$ is an element of the basis of $\boldsymbol{V}_{0 h}^{r}$ and where $\boldsymbol{\varphi}_{0 j}^{d}$ is an element of the basis of $\boldsymbol{V}_{0 h}^{d}$.

These terms are classically evaluated by summing elements contributions. However, one may have particular situations where coarse elements are crossed by the coupling zone boundary and the crack. For each of these particular elements, one has to create an integration mesh that fits both the parts of the coupling zone boundary and the crack, contained in this element, as shown in figure 10, in order to use classical quatrature formulae in this integration mesh.

Before giving some numerical results, let us observe that system 37 is similar to systems derived by domain decomposition methods. It can then be solved by Krylov solvers. Very recently, the FETI method [21] has been adapted to the solution of such systems [17] and proved to be very efficient. This efficiency is due in particular to the fact that thanks to the energy coupling operator involved by the Arlequin formulation, it is possible de derive "natural" local pre-conditioners (see [17], for details).

\section{$5 \quad$ Numerical results}

Three tests are considered in this Section to assess the methodology developed in this paper. For the three of them, we have taken $\alpha_{0}=0$ in the coupling zone and $\alpha_{0}=10^{-3}$, in the free zone.

The first involves a 2D-structure with a kinked crack; the kink being very close to the crak tip. Our results are compared to the ones obtained with a classical globally refined finite element model. The propagation of a crack in 

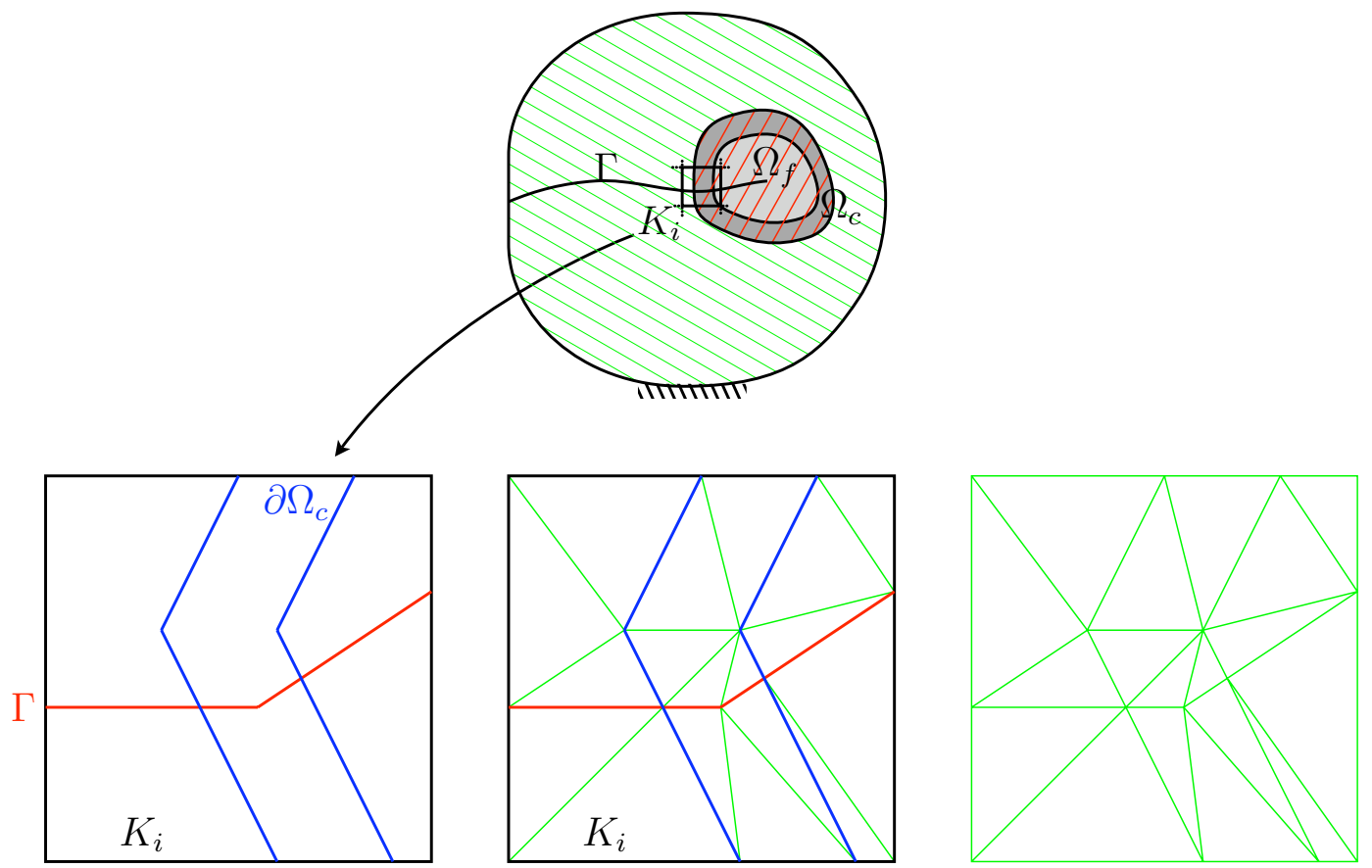

Fig. 10. Mesh subdivision for the integration issue

an elastic beam is studied as a second test and our results are compared to those obtained in [6]. The third result shows the capability of the method to propagate a crack from a side to the opposite one of a square plate. Let us observe here that for all of the tests, there is no compatibility requirement between the coarse and fine meshes.

\subsection{A kinked crack}

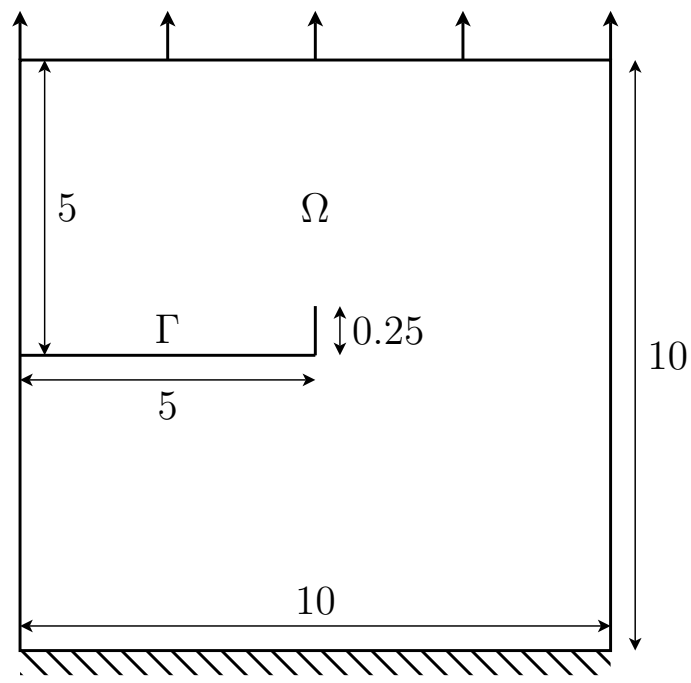

Fig. 11. kinked crack problem 
We consider a homogeneous and isotropic cracked elastic plate in plane strain conditions. The dimensions of the plate (in meters) are given in figure 11. The Young's modulus is equal to $210^{5} \mathrm{MPa}$ and the coefficient of Poisson is equal to 0.3 . The plate is clamped on its lower edge. A given normal displacement is prescribed on the upper edge. The two other edges are free. A straight crack is deviated sharply with a relatively small increment as shown by figure 11 . This situation may represent the deviations of a crack during its propagation with small and unknown increments. For the numerical simulation of this test, the two interacting singularities have to be considered, either by introducing the appropiate singular modes by XFEM, for instance, or, as done here, by refining sufficiently the local domain mesh around the two singular points.

For comparison purpose, we have first computed a classical globally refined monomodel finite element solution. The mesh size in the near crack tip is taken equal to about $0.02 \mathrm{~m}$ in the very near crack tip zone. The deformed mesh is shown in figure 12 .

Three cases are computed with our methodology.

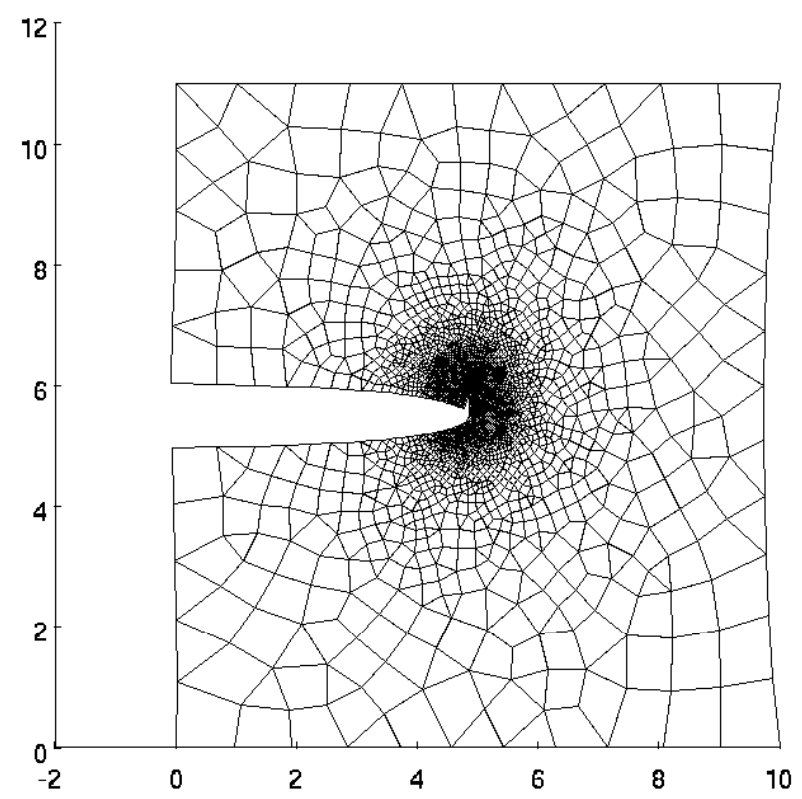

Fig. 12. Classical globally refined monomodel finite element solution

In the first, a uniform quadrangular $9 \times 9$ elements fixed mesh is used for the global domain. The edges of this mesh do not match the crack. The patch is a disk of radius $r=1.2 \mathrm{~m}$, centered at the crack tip. The coarse element, of diameter $H_{c}=1.73 m$, containing the two hot points (the kink and the crack tip) is included in the free zone. The thickness of the coupling zone is taken equal to $r / 8$ and the mesh size of the patch in the very near crack tip zone is of about $0.02 \mathrm{~m}$.

The deformed meshes are given in the left part of figure 13. A zoom of the 
critical zone is shown in the right part of this figure. The red lines represent the position of the deformed faces of the crack in the global model. One can observe that while the coarse deformed crack does fit the fine crack in the coupling zone, the two surfaces do not match in the free zone. But, as mentioned at the end of Subsection 4.2, this is with no impact on the Arlequin solution, dominated by the fine local solution in this zone thanks to the fact that the fine model is stressed in this zone by choosing its weight parameter $\alpha_{1}$ nearly equal to 1.

Figure 14 shows the Von Mises stresses at the Gauss quadrature points of the patch and one can notice that the two singularities are captured (up to the precision of the used numerical models: no h-adaptivity is herein carried out).
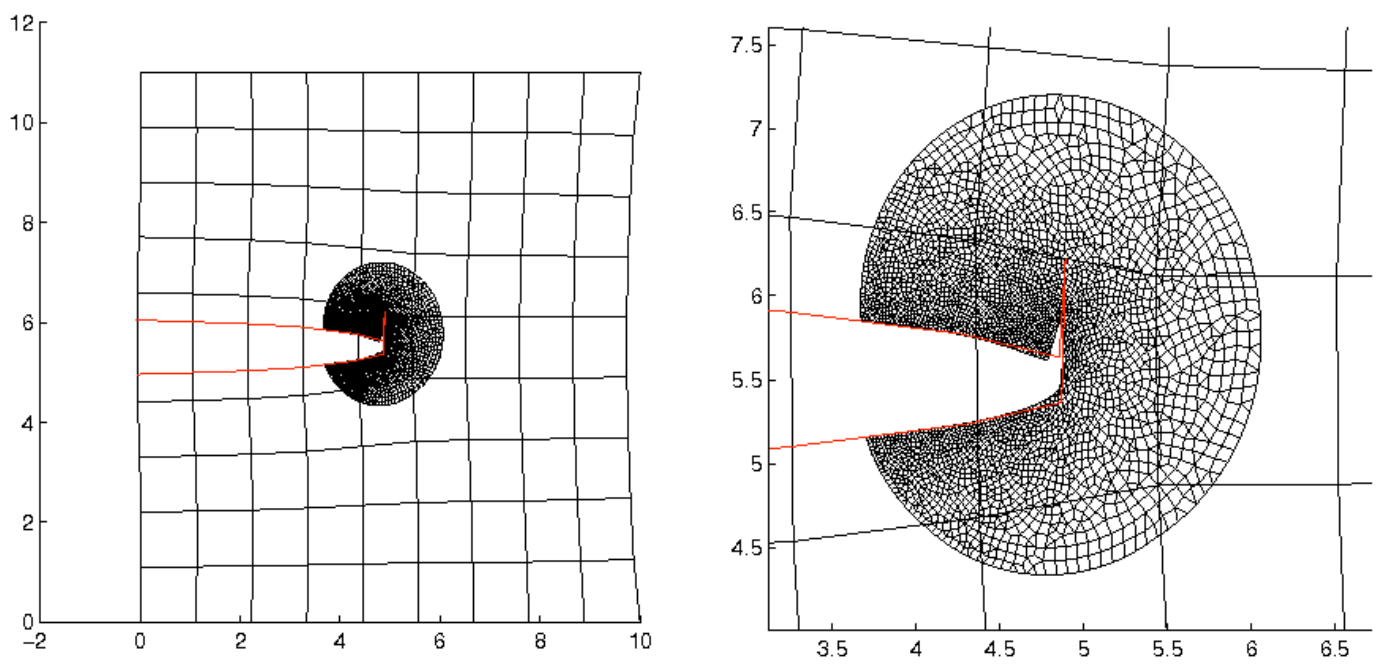

Fig. 13. deformed meshes of the substrate and the patch
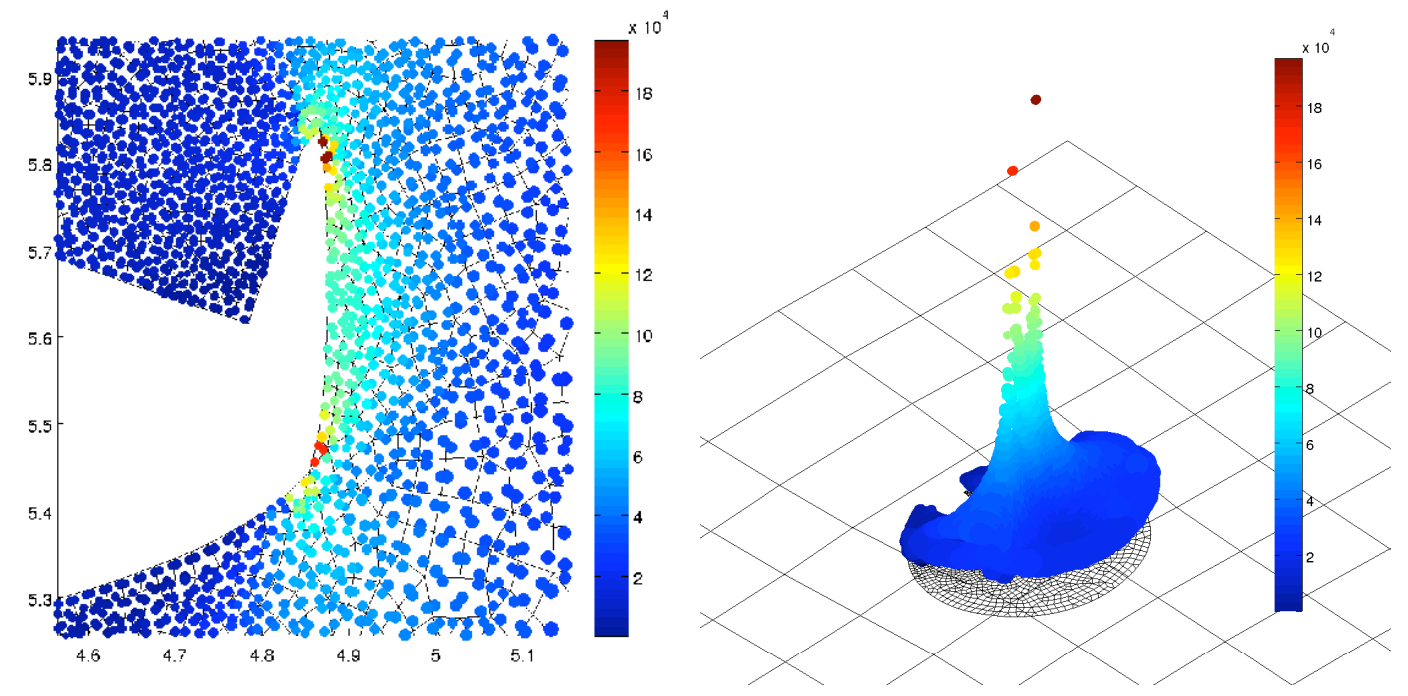

Fig. 14. interaction between the sigularities on the patch mesh 

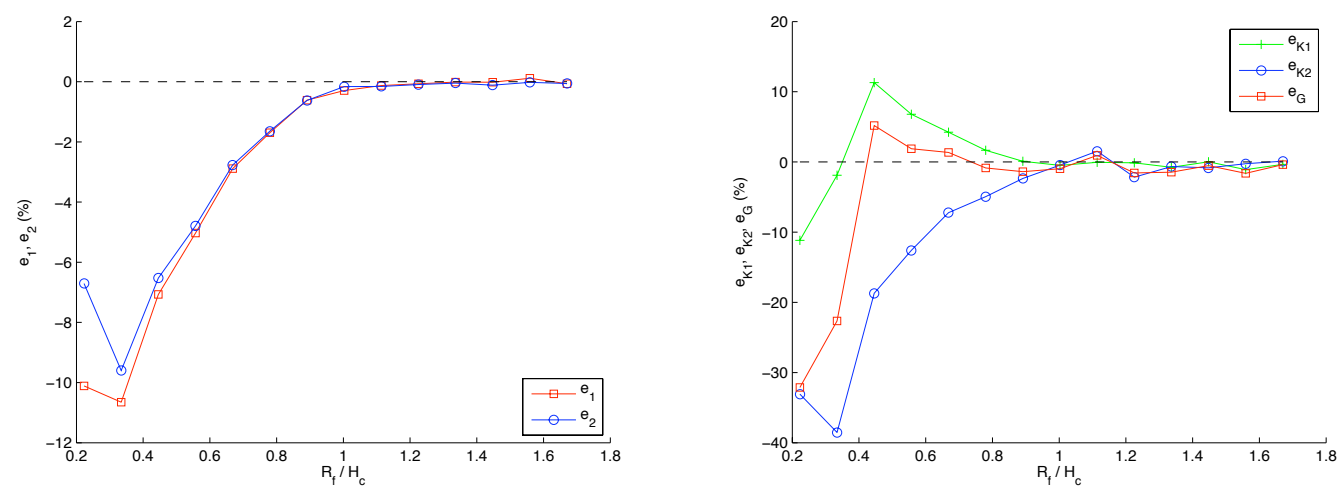

Fig. 15. Evolution of the error on chosen mechanical quantities of interest with the increase of the radius of the free zone and with a fixed ratio of patch radius vs. free zone radius

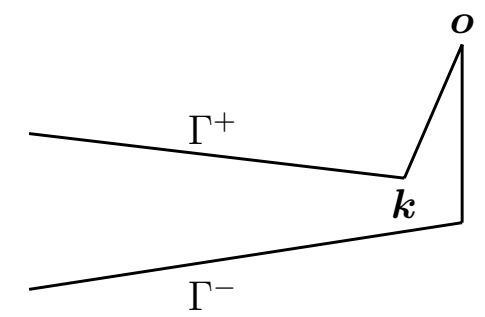

Fig. 16. nodes of interest

The similarity of the reference solution given in figure 12 with the one shown in figure 13 is clear.

For further comparison, stress intensity factors $K I$ and $K I I$ and energy release rates $G$ are computed for the considered reference monomodel and for Arlequin-XFEM models with patches having radii varying from $0.2 \mathrm{~m}$ to $3 \mathrm{~m}$, with 0.125 ratio between the thickness of the coupling zone and the radius of the patches. The right part of figure 15 shows a reasonable decrease, followed by a stabilisation of the error with the increase of the ratio between the the radius of the free zone, denoted by $R_{f}$, and the coarse mesh size, denoted by $H_{c}$. Notice that the relative error is already less than $5 \%$ when $\frac{R_{f}}{H_{c}}$ is greater than 0.8 and of order $1 \%$ when this ratio is of order 1 . The left part of figure 15 shows the same tendancy for local relative displacement errors $e_{1}=\frac{\left(u_{y}(\boldsymbol{o})-u_{y}^{r e f}(\boldsymbol{o})\right)}{u_{y}^{r e f}(\boldsymbol{o})} \times 100$ and $e_{2}=\frac{\left(u_{y}(\boldsymbol{k})-u_{y}^{r e f}(\boldsymbol{k})\right)}{u_{y}^{r e f}(\boldsymbol{k})} \times 100$, at the two critical points $\boldsymbol{O}$ and $\boldsymbol{k}$, shown in figure 16. One can also notice significant errors when the radius of the free zone is too small with respect to $H_{c}$. In figure 17 , we show the deformed meshes for such an inappropriate choice of the free zone dimension. These results are to be linked to hypothesis (30).

In a second case, the same procedure has been used by changing only the coarse mesh size: a $19 \times 19$ coarse structured mesh is used instead of the $9 \times 9$ mesh. 


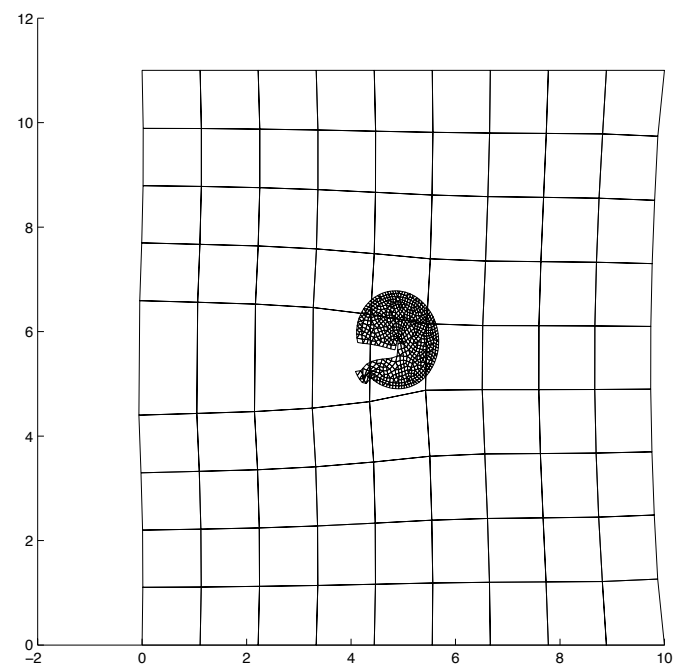

Fig. 17. Deformed meshes for inappropriate choice of the free zone dimension

In this case, the diameter of the coarse elements is $H_{c}=0.74 m$. The obtained results are shown in figure 18. A remarkable feature is the similarity of the profile of error between the two considered numerical models. For instance, for both models, the error in terms of stress intensity factors and energy release rate, is of order $1 \%$ when the ratio $\frac{R_{f}}{H_{c}}$ is of order 1 .
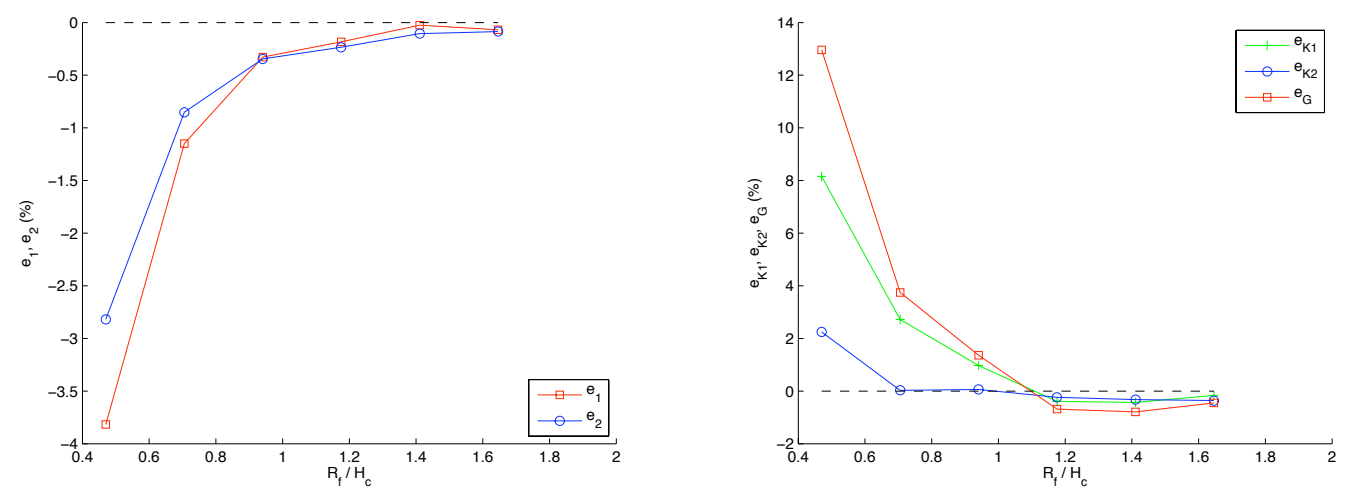

Fig. 18. Evolution of the error on chosen mechanical quantities of interest with the increase of the radius of the free zone and with a fixed ratio of patch radius vs. free zone radius

In a third case, the uniform quadrangular $19 \times 19$ elements fixed mesh is used for the global domain, as in the second case. But in this third case, the patch is a disk of a fixed radius $r=1.5 \mathrm{~m}$, centered at the crack tip. It is meshed finely as in the first test. We have considered various thiknesses of the coupling zone which induce various radii $R_{f}$ for the free zones, while keeping fixed the patch mesh. The same comparisons in terms of the same quantities of interest 
as for the first and second tests are reported in figure 19.
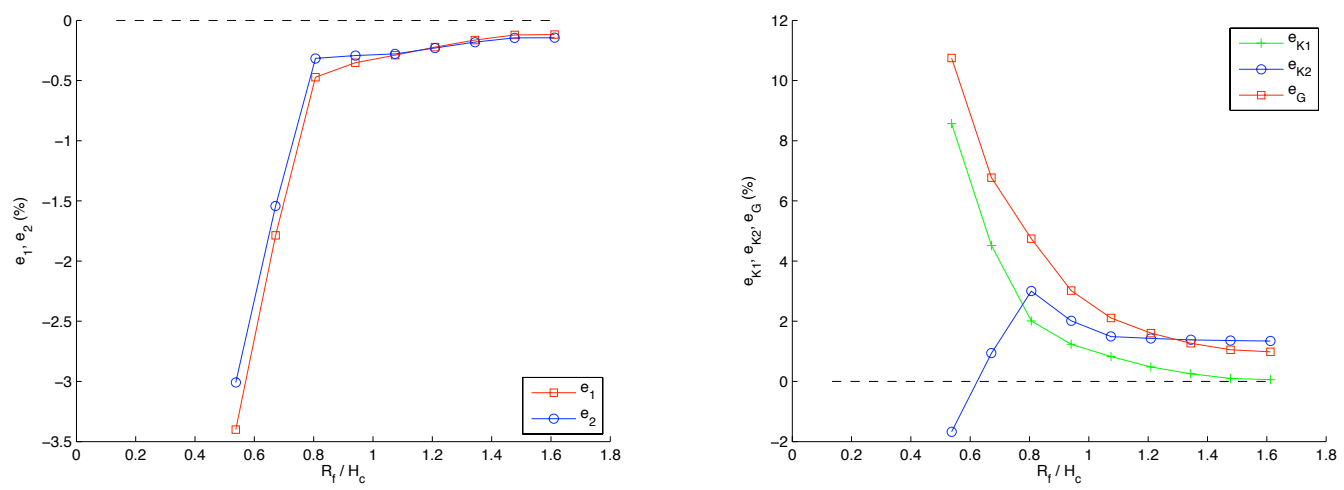

Fig. 19. Evolution of the error on chosen mechanical quantities of interest with the increase of the radius of the free zone and fixed patch radius

One can observe the same tendancy, with more regular decrease of the errors with the increase of $\frac{R_{f}}{H_{c}}$. This can be explained by the fact that in the considered case, the fine local mesh is kept fixed, while it is not in the first and second ones.

\subsection{The double-cantilever beam test}

The test studied in [6] and shown in the introduction of the present paper is analyzed here by our new methodology. It consists in a rectangular $L \times h 2 D$ homegeneous and isotropic elastic structure in plane stress conditions, with $L=0.3 \mathrm{~m}$ and $h=0.1 \mathrm{~m}$. The Young modulus is equal to $210^{5} \mathrm{MPa}$ and the Poisson coefficient is equal to 0.3 . The structure contains an initial straight crack of lenght $a=0.1 \mathrm{~m}$, aligned with the $x$ axis. A small increment of $0.002 \mathrm{~m}$ with an angle $\theta$ of $5.71^{\circ}$ is added to the initial crack and the structure is submitted to a symmetric pointwise opening loading $P=877 \mathrm{~N}$, as shown in figure 20. We study the quasi-static growth of the crack. The used procedure is the following: the stress intensity factors using the domain form of the interaction integrals are computed. With these quantities, the propagation's angle, corresponding to the maximum orthoradial stress $\sigma_{\theta \theta}$ is evaluated and the crack is advanced $0.002 \mathrm{~m}$ in this direction.

Figure 21 shows the propagation of the crack which is qualitatively similar to the one obtained in [6]. Figure 22 shows zooms of the crack tip zone at the last step of the propagation. The deformed meshes are given by the left part of the figure and the Von Mises stresses in the quadrature points of the patch's mesh by the right part. 


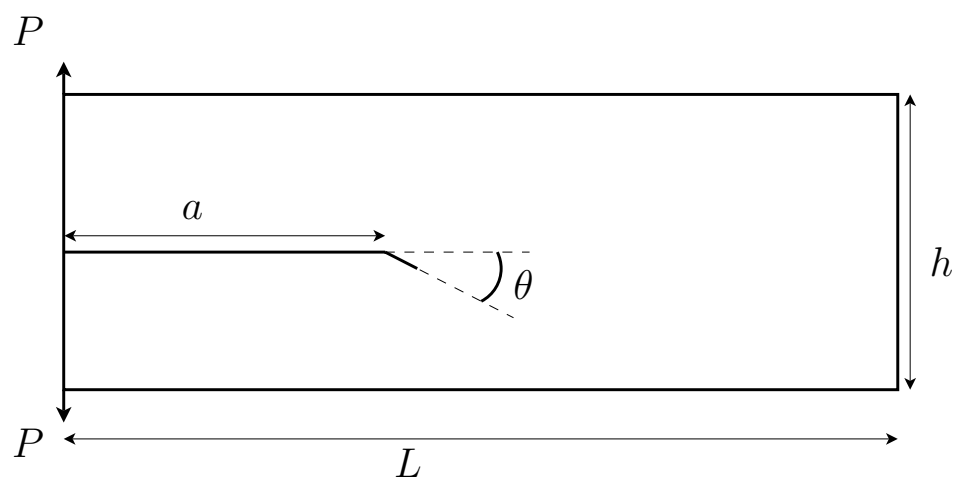

Fig. 20. Geometry of the double cantilever beam test
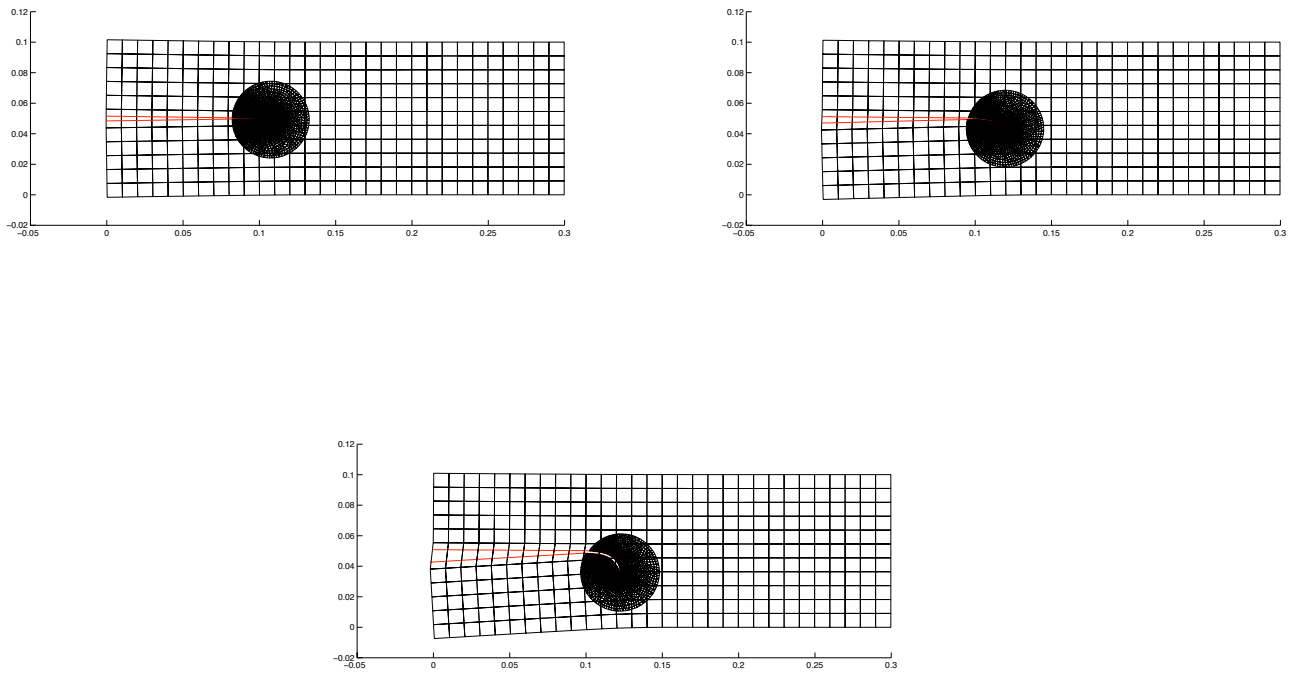

Fig. 21. Deformed meshes of the substrate and the patch
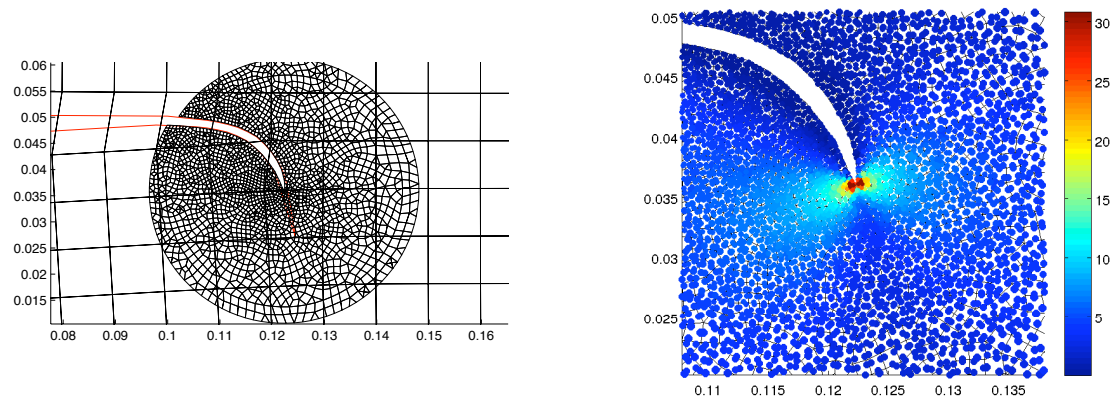

Fig. 22. Zoom of the near crack tip zone

5.3 From a side-to-another propagation of a crack in a square plate

In this last test, we consider a square homegeneous and isotropic elastic plate in plane strain conditions, with an initial relatively small crack. The dimensions 
of the plate are given in figure 23. The Young modulus is equal to $210^{5} \mathrm{MPa}$ and the Poisson coefficient is equal to 0.3. The initial straight crack of lenght $0.1 \mathrm{~m}$ is aligned with the $x$ axis. The plate is clamped on its lower edge and submitted to opening loads on its upper edge. The reminder of the boundary is free. A coarse and fixed uniform 9X9 bilinear finite element space is used for

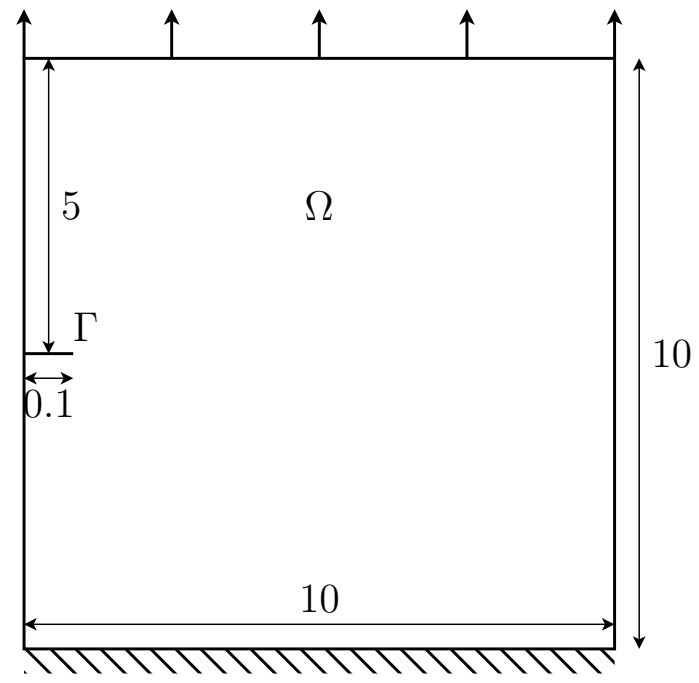

Fig. 23. mode1 propagation problem

the approximation of the coarse Arlequin solution. When completely included in the global domain, the radius of the considered (whole) patch is equal to $1.5 \mathrm{~m}$ and the thickness of the coupling zone is equal to $0.2 \mathrm{~m}$. The mesh size of the patch, in the near crack tip zone, is taken equal to $0.01 \mathrm{~m}$.

The patch geometry is adapted whenever it touches the boundary of the plate. This is simply realized by considering the restriction of the whole patch to the global domain. Observe then that the coupling zone is no more the crown satifying the condition (16), but the portion of the crown, satisfying the following adapted condition:

$$
\partial \Omega_{1} \cap \Omega_{0} \subset \partial \Omega_{c}
$$

The same procedure as the one used in the previous subsection is used for the quasi-static propagation of the crack. Four snapshots of the deformed meshes during the propagation of the crack are represented in figure 24. A zoom of the crack tip zone in the first opening step is given in figure 25. This test could possibly be delicate to achieve with classical enrichment methods.

\section{Conclusion}

A new methodology using the Heaviside enrichment key feature of the extendend finite element method within the Arlequin framework has been developed 

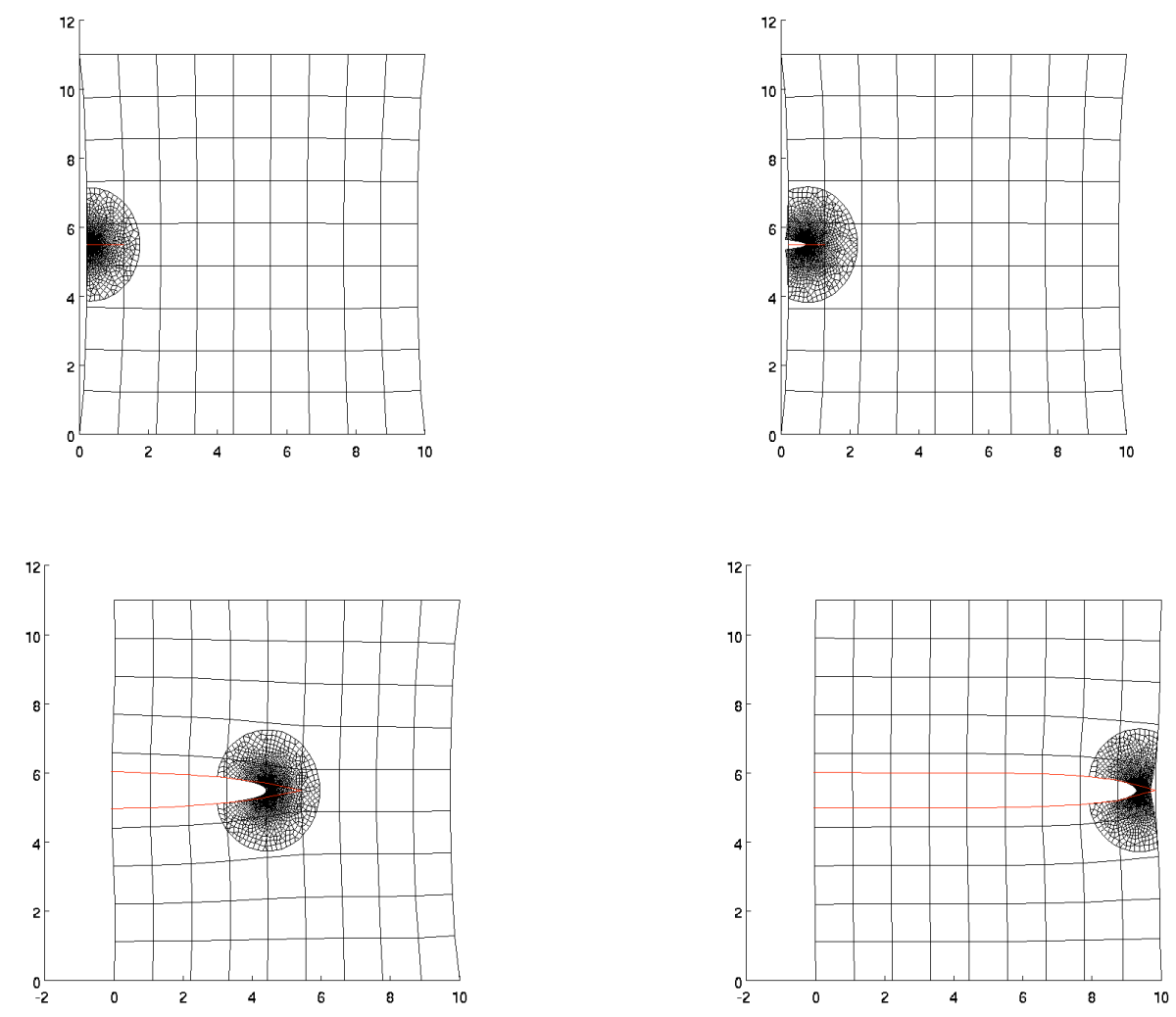

Fig. 24. Deformed meshes of the substrate and the patch

in this paper for flexible simulations of kinked cracks and the propagation of cracks. When compared to our previous works (e.g. [11]), the new methodology reduces significantly the global computational costs since a small cracked patch is used in the working zone; the part of the crack located outside of the critical zones being taken into account by means of a Heaviside function.

For the propagation of a crack, the patch, finely meshed, is attached to the crack tip and moves with it over the underlying global domain, approximated by a fixed coarse finite element enriched by a Heaviside function.

This methodology is assessed in this paper by some simple but relevant mecanical tests. A kinked crack is calculated by following two different scenarii. For both of these scenarii, reasonable low errors, in terms of quantities of interst, are reached when the size of the free zone is of the same order than the size of the coarse elements containing the singularities. The potential of the methodology to propagate cracks with significantly enhanced flexibility is shown by firstly investigating a double cantilever test without using a priori knowledge of the local singular modes. Our results are shown to compare qualitatively well with the ones given by Belytschko and Black [6]. Secondly, a final feasibility test is carried out to show the capapility of the method to propagate a crack from a side to another of a structure by adaptig the patch geometry. The extension of this methodology to the simulation of the propagation of 


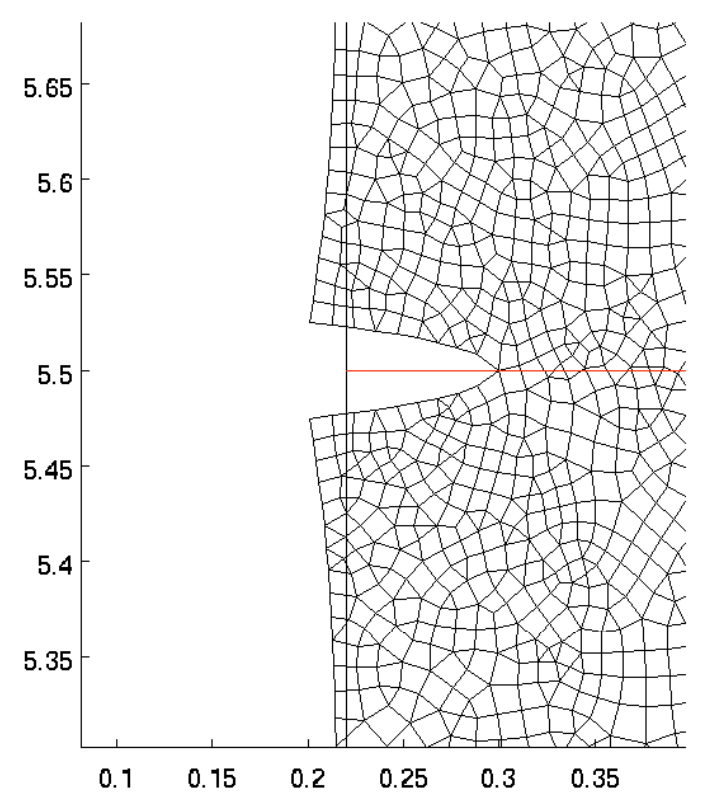

Fig. 25. zoom on the initial crack at the beginning of the propagation cracks, while taking into account more complex physics in the working zones, is an ongoing work.

\section{References}

[1] R. Adams, Sobolev Spaces, Academic Press, New York, 1976.

[2] I. Babuška, J.-M. Melenk, The partition of unity method, Int. J. Numer. Meth. Engrg. 40 (1997) 727-758.

[3] P.T. Bauman, H. Ben Dhia, N. Elkhodja, J.T. Oden, S. Prudhomme, On the application of the Arlequin method to the coupling of particle and continuum models, Comput. Mech. 42 (2008) 511-530.

[4] I. Babuška, T. Strouboulis, K. Copps, The design and analysis of the Generalized Finite Element Method, Comp. Meth. Appl. Mech. Engrg. 181 (2000) 43-69.

[5] T. Belytschko, YY. Lu, L. GU, Element-free Galerkin methods, Int. J. Numer. Meth.Engrg. 37 (1994) 229-256.

[6] T. Belytschko, T. Black, Elastic crack growth in finite elements with minimal remeshing Int. J. Numer. Meth. Engrg. 45 (1999) 601-620.

[7] T. Belytschko, N. Moës, S. Usui, C. Parimi, Arbitrary discontinuities in finite elements, Int. J. Numer. Meth. Engrg. 50 (2001) 993-1013. 
[8] H. Ben Dhia, Multiscale mechanical problems: the Arlequin method, C. R. Acad. Sci. Série IIb, 326 (1998) 899-904.

[9] H. Ben Dhia, G. Rateau, Mathematical analysis of the mixed Arlequin method, C. R. Acad. Sci. Paris Série I. 332 (2001) 649-654.

[10] H. Ben Dhia, G. Rateau, Application of the Arlequin method to some structures with defects, Finte Element European Review, 11 (2002) 291-304.

[11] H. Ben Dhia, G. Rateau, The Arlequin method as a flexible engineering design tool, Int. J. Numer. Meth.Engrg. 62 (2005) 1442-1462.

[12] H. Ben Dhia, Global-local approaches: the Arlequin framework, European Journal of Computational Mechanics, 15 (2006) 67-80.

[13] H. Ben Dhia, The Arlequin method: a partition of models for concurrent multiscale analyses, Challenges in Computational Mechanics Workshop, 10-12 May, 2006, Cachan, France, (2006).

[14] H. Ben Dhia, C. Zammali, Level-Sets, placement and velocity-based lagrangian formulation of impact problems, Int. J. Numer. Meth. Engrg. 69 (2007) 27112735 .

[15] H. Ben Dhia, N. Elkhodja, Coupling of atomistic and continuum models in the Arlequin framework, Proceedings of the $8^{\text {eme }}$ Congrès de Mécanique, El Jadida, Maroc, April 17-20, 133-135, (2007).

[16] H. Ben Dhia, Further insigths by theoretical investigations of the multiscale Arlequin method, Int. J. Multi. Comp. Engrg. 6 (2008) 215-232.

[17] H. Ben Dhia, N. Elkhodja, F.-X Roux, Multi-modeling of multi-alterated structures in the Arlequin framework. Solution with a domain decomposition solver, Eur. J. of Comp. Mech. 17 (2008) 969-980.

[18] E. Chahine, P. Laborde, Y Renard, Crack tip enrichment in the XFEM method using a cut-off function, Int. J. Numer. Meth. Engng. 75 (2008) 629-646.

[19] P.G. Ciarlet, The Finite Element Method for Elliptic Problems, North-Holland, (1978).

[20] J. Dolbow, N. Moës, T. Belytschko, Discontinuous enrichment in finite element with a partition of unity method, Finite Elements in Analysis and Design. 36 (2000) 235-260.

[21] C. Farhat, F.-X. Roux, A method of finite element tearing and interconnecting and its parallel solution algorithm, Int. J. Numer. Meth. Engng. 32 (1991) 12051227.

[22] J. Fish, The s-version of the finite element method, Computer and Structures, 43 (1992) 539-547.

[23] R. Glowinski, J. He, J. Rappaz, J. Wagner, Approximation of multi-scale elliptic problems using patches of finite elements, C.R. Acad Sci. Paris, Ser.I. 337 (2003) $779-684$. 
[24] R. Glowinski, J. He, A. Lozinski, J. Rappaz, J. Wagner, Finite element approximation of multi-scale elliptic problems using patches of elements, Numer. Math. 101 (2005) 663-687.

[25] T.J.R. Hughes, The Finite Element Method: Linear Static and Dynamic Finite Element Analysis, Englewood-Cliffs (Prentice Hall), (1987).

[26] P. Laborde, J. Pommier, Y. Renard, M. Salaün, High order extended finite element method for cracked domains, Int. J. Numer. Meth. Engng. 64 (2005) 354-381.

[27] S.F. McCormick, J. Thomas, The fast adaptive composite-grid (FAC) method for elliptic equations, Math. Comp. 46 (174) (1986) 439-456.

[28] J.-M. Melenk, I. Babuška, The partition of unity finite element method: Basic theory and applications, Comp. Meth. Appl. Mech. Engrg. 139 (1996) 289-314.

[29] N. Moës, J. Dolbow, T. Belytschko, A finite element method for crack growth without remeshing, Int. J. Numer. Meth. Engrg. 46 (1999) 131-150.

[30] B. Nayroles, G. Touzot, P. Villon, Generalizing the finite element method: diffuse approximation and diffuse elements, Computers and Structures, 10 (1992) 307-318.

[31] B. Prabel, S. Marie, A. Combescure, Using the XFEM method to model the dynamic propagation and arrest of cleavage cracks in ferritics steel, Engineering Fracture Mechanics. 75 (2000) 2984-3009.

[32] S. Prudhomme, L. Chamoin, H. Ben Dhia, P.T. Bauman, An adaptive strategy for the control of modeling error in two-dimensional atomic-to-continuum coupling simulations, Comp. Meth. Appl. Mech. Engrg., 198 (2009) 1887-1901.

[33] G. Rateau, Méthode Arlequin pour les Problèmes Mécaniques Multi-échelles. Application à des Problèmes de Jonction et de Fissuration de Structures Élancées, PhD Thesis, Ecole Centrale PARIS, (2003).

[34] G. Rateau, H. Ben Dhia, Méthode Arlequin pour la propagation de défauts dans des pneus : Etude de Faisabilité, Post Doctorat Report for Michelin Society, (2005).

[35] Y. Romdhane, H. Ben Dhia, Michelin Society Report, 2006.

[36] H.A. Schwarz, ber einen Grenzbergang durch alternierendes Verfahren, Vierteljahrsschrift der Naturforschenden Gesellschaft in Zrich 15 (1870) 272286

[37] J.L. Steger, J.A. Benek. On the use of composite grid schemes in computational aerodynamics, Comput. Meth. Appl. Mech. Engrg. 64 (1987) 301-320.

[38] S.P. Xiao, T. Belytschko, A bridging domain method for coupling continua with molecular dynamics, Comp. Meth. Appl. Mech. Engrg. 193 (2004) 1645-1669.

[39] O.C. Zienkiewicz, R.L.G. Taylor, The Finite Element Method, 1, 2, McGrawHill, New York, (1999), (2001). 